\title{
Fractional Kinetic Equations Associated with Incomplete $I$-Functions
}

\author{
Manish Kumar Bansal ${ }^{1}\left(\mathbb{D}\right.$, Devendra Kumar ${ }^{2, *} \mathbb{\infty}$, Priyanka Harjule ${ }^{3}$ and Jagdev Singh ${ }^{4}(\mathbb{C}$ \\ 1 Department of Applied Sciences, Government Engineering College, Banswara 327001, Rajasthan, India; \\ bansalmanish443@gmail.com \\ 2 Department of Mathematics, University of Rajasthan, Jaipur 302004, Rajasthan, India \\ 3 Department of Mathematics, IIIT, Kota, MNIT Campus, Jaipur 302017, Rajasthan, India; \\ priyanka.maths@iiitkota.ac.in \\ 4 Department of Mathematics, JECRC University, Jaipur 303905, Rajasthan, India; \\ jagdevsinghrathore@gmail.com \\ * Correspondence: devendra.maths@gmail.com
}

Received: 12 April 2020; Accepted: 30 April 2020; Published: 4 May 2020

\begin{abstract}
In this paper, we investigate the solution of fractional kinetic equation (FKE) associated with the incomplete I-function (IIF) by using the well-known integral transform (Laplace transform). The FKE plays a great role in solving astrophysical problems. The solutions are represented in terms of IIF. Next, we present some interesting corollaries by specializing the parameters of IIF in the form of simpler special functions and also mention a few known results, which are very useful in solving physical or real-life problems. Finally, some graphical results are presented to demonstrate the influence of the order of the fractional integral operator on the reaction rate.
\end{abstract}

Keywords: fractional kinetic equation; Riemann-Liouville fractional integral operator; incomplete I-functions; Laplace transform

\section{Introduction}

Arbitrary-order calculus (AOC) is a useful mathematical device that enables the study of arbitrary-order integrals and derivatives [1-4]. Its origin dates back to the 1695 letter from Leibniz to L'Hôpital. The noble developments in the field of fractional-order calculus (FOC) in relevant conceptual research and in solving real-time problems have been extensively studied comparatively recently. The pioneering contributions in fractional calculus were given by legendary mathematicians viz. Euler, Fourier, Abel, Liouville, or Riemann. For explicit knowledge of arbitrary-order derivatives and integrals, one can refer to [5] and the references therein. The intellect of fractional derivative equations (FDEs) along with their implications have had a significant impact on various science and engineering systems. In particular, the kinetic equations (KEs) characterize the relationship between concentrations of the materials and time. KE is applied in gas theory, plasma physics, aerodynamics, etc. The solution of KE gives the distribution function of the dynamical states of a single particle, which often depends on the coordinates, time, and velocity. The expansions and generic nature of arbitrary-order kinetic equations associated with the fractional-order operators was well established in [6-9]. Since the last few decades, fractional kinetic equations in several shapes and configurations have been widely and productively employed in describing various significant problems of physics and astrophysics (see the recent papers [10-17]). 
The FDE describing the rates at which the reaction, destruction, and production change was determined by Haubold and Mathai [6], which is presented in the following equation:

$$
\frac{d \Theta}{d w}=-d\left(\Theta_{w}\right)+p\left(\Theta_{w}\right),
$$

where $\Theta=\Theta(w)$ gives the reaction rate, $d=d(\Theta)$ gives the destruction rate, $\mathrm{p}=\mathrm{p}(\Theta)$ is the production rate, and $\Theta_{w}$ represents the function defined by $\Theta_{w}\left(w^{*}\right)=\Theta\left(w-w^{*}\right), w^{*}>0$.

Now, if the spatial fluctuation and the inhomogeneities in the quantity $\Theta(w)$ are ignored, then (1) is converted into:

$$
\frac{d \Theta_{i}}{d \mathrm{w}}=-\mathrm{c}_{\mathrm{i}} \Theta_{i}(\mathrm{w})
$$

subject to the initial condition that $\Theta_{i}(w=0)=\Theta_{0}$ is the number density of species iat initial time $(w=0), c_{i}>0$.

Equation (2) can be written after integrating:

$$
\Theta(\mathrm{w})-\Theta_{0}=-\mathrm{c}_{0} D_{\mathrm{w}}^{-1} \Theta(\mathrm{w}),
$$

where $D_{\mathrm{w}}^{-1}$ is known as the integral operator.

Haubold and Mathai [6] gave the extension of Equation (3) (known as the fractional kinetic equation (FKE)) as follows:

$$
\Theta(w)-\Theta_{0}=-c^{\beta}{ }_{0} D_{w}^{-\beta} \Theta(w),
$$

where $D_{\mathrm{w}}^{-\beta}$ denotes the familiar Riemann-Liouville fractional integral operator.

The solution of FKE (4) is given below:

$$
\Theta(\mathrm{w})=\Theta_{0} \sum_{\kappa=0}^{\infty} \frac{(-1)^{\kappa}}{\Gamma(\beta \kappa+1)}(\mathrm{cw})^{\beta \kappa} .
$$

From the perspective of the effectiveness and great significance of the KE in many physics and astrophysical problems, we established a solution of FKE involving the IIF.

The very familiar gamma function $\Gamma(s)$ is defined as follows:

$$
\Gamma(\Im)=\left\{\begin{array}{lc}
\int_{0}^{\infty} e^{-u} u^{\Im-1} d u, & (\Re(\Im)>0) \\
\frac{\Gamma(\Im+\mathfrak{K})}{(\Im)_{\mathfrak{K}}}, & \left(\Im \in \mathbb{C} \backslash \mathbb{Z}_{0}^{-} ; \mathfrak{K} \in \mathbb{N}_{0}\right),
\end{array}\right.
$$

where $(\Im)_{\mathfrak{K}}$ denotes the Pochhammer symbol defined (for $\Im \in \mathbb{C}$ and $\mathfrak{K} \in \mathbb{N}_{0}$ ) by:

$$
(\Im)_{\mathfrak{K}}=\frac{\Gamma(\Im+\mathfrak{K})}{\Gamma(\Im)}= \begin{cases}1, & (\mathfrak{K}=0 ; \Im \in \mathbb{C} \backslash\{0\}) \\ \Im(\Im+1) \cdots(\Im+s-1), & (\mathfrak{K}=s \in \mathbb{N} ; \Im \in \mathbb{C}),\end{cases}
$$

provided that the gamma quotient exists.

The incomplete gamma functions (IGFs) $\gamma(\Im, x)$ and $\Gamma(\Im, x)$ are presented in the following manner:

$$
\gamma(\Im, x)=\int_{0}^{x} u^{\Im-1} e^{-u} d u, \quad(\Re(\Im)>0 ; x \geqq 0),
$$


and:

$$
\Gamma(\Im, x)=\int_{x}^{\infty} u^{\Im-1} e^{-u} d u, \quad(x \geqq 0 ; \Re(\Im)>0 \text { when } x=0),
$$

respectively, which satisfy the subsequent decomposition formula:

$$
\gamma(\Im, x)+\Gamma(\Im, x)=\Gamma(\Im), \quad(\Re(\Im)>0) .
$$

The gamma function $\Gamma(\Im)$ and IGFs $\gamma(\Im, x)$ and $\Gamma(\Im, x)$, which is defined in (6), (8), and (9), respectively, play the main role in the field of communication theory, probability theory, groundwater pumping modeling, quantum physics, mathematical physics, statistics, solid state physics, engineering, and science (see, for example, [18,19]; see also the recent papers [20-28]).

Recently, Bansal and Kumar ([29], p. 1248, Equations (1.6)-(1.9)) defined the incomplete I-functions (Г) $I_{p_{i}, q_{i}, r}^{m, n}(z)$ and ${ }^{(\gamma)} I_{p_{i}, q_{i}, r}^{m, n}(z)$ associated with the IGFs $\gamma(\Im, x)$ and $\Gamma(\Im, x)$ as follows:

$$
\begin{aligned}
{ }^{(\Gamma)} I_{p_{\ell, q_{\ell}, r}}^{m, n}(z) & ={ }^{(\Gamma)} I_{p_{\ell}, q_{\ell}, r}^{m, n}\left[z \mid \begin{array}{c}
\left(e_{1}, E_{1}, x\right),\left(e_{j}, E_{j}\right)_{2, n},\left(e_{j \ell}, E_{j \ell}\right)_{n+1, p_{\ell}} \\
\left(f_{j}, F_{j}\right)_{1, m},\left(f_{j \ell}, F_{j \ell}\right)_{m+1, q_{\ell}}
\end{array}\right] \\
& =\frac{1}{2 \pi i} \int_{\mathfrak{L}} \mathbb{K}(\xi, x) z^{-\xi} d \xi
\end{aligned}
$$

for all $z \neq 0$; here, $i=\sqrt{-1}$ and:

$$
\mathbb{K}(\xi, x)=\frac{\Gamma\left(1-e_{1}-E_{1} \xi, x\right) \prod_{j=1}^{m} \Gamma\left(f_{j}+F_{j} \xi\right) \prod_{j=2}^{n} \Gamma\left(1-e_{j}-E_{j} \xi\right)}{\sum_{\ell=1}^{r}\left[\prod_{j=m+1}^{q_{\ell}} \Gamma\left(1-f_{j \ell}-F_{j \ell} \xi\right) \prod_{j=n+1}^{p_{\ell}} \Gamma\left(e_{j \ell}+E_{j \ell} \xi\right)\right]}
$$

and:

$$
\begin{aligned}
{ }^{(\gamma)} I_{p_{\ell}, q_{\ell}, r}^{m, n}(z) & ={ }^{(\gamma)} I_{p_{\ell}, q_{\ell}, r}^{m, n}\left[z \mid \begin{array}{c}
\left(e_{1}, E_{1}, x\right),\left(e_{j}, E_{j}\right)_{2, n},\left(e_{j \ell}, E_{j \ell}\right)_{n+1, p_{\ell}} \\
\left(f_{j}, F_{j}\right)_{1, m},\left(f_{j \ell}, F_{j \ell}\right)_{m+1, q_{\ell}}
\end{array}\right] \\
& =\frac{1}{2 \pi i} \int_{\mathfrak{L}} \mathbb{L}(\xi, x) z^{-\xi} d \xi
\end{aligned}
$$

for all $z \neq 0$; here, $i=\sqrt{-1}$ and:

$$
\mathbb{L}(\xi, x)=\frac{\gamma\left(1-e_{1}-E_{1} \xi, x\right) \prod_{j=1}^{m} \Gamma\left(f_{j}+F_{j} \xi\right) \prod_{j=2}^{n} \Gamma\left(1-e_{j}-E_{j} \xi\right)}{\sum_{\ell=1}^{r}\left[\prod_{j=m+1}^{q_{\ell}} \Gamma\left(1-f_{j \ell}-F_{j \ell} \xi\right) \prod_{j=n+1}^{p_{\ell}} \Gamma\left(e_{j \ell}+E_{j \ell} \xi\right)\right]} .
$$

The incomplete $I$-functions ${ }^{(\Gamma)} I_{p_{\ell, q_{\ell}, r}}^{m, n}(z)$ and ${ }^{(\gamma)} I_{p_{\ell, q_{\ell}, r}}^{m, n}(z)$ in (11) and (13) exist for all $x \geq 0$ under the set of conditions as mentioned below.

The contour $\mathfrak{L}$ in the complex $\xi$-plane extends from $\gamma-i \infty$ to $\gamma+i \infty, \gamma \in \mathbb{R}$, and poles of the gamma functions $\Gamma\left(1-e_{j}-E_{j} \xi\right), j=\overline{1, n}$ do not exactly match with the poles of the gamma functions $\Gamma\left(f_{j}+F_{j} \xi\right), j=\overline{1, m}$. The parameters $m, n, p_{\ell}, q_{\ell}$ are non-negative integers satisfying $0 \leq n \leq p_{\ell}$, $0 \leq m \leq q_{\ell}$ for $i=\overline{1, r}$. The parameters $E_{j}, F_{j}, E_{j \ell}, F_{j \ell}$ are positive numbers, and $e_{j}, f_{j}, e_{j \ell}, f_{j \ell}$ are 
complex. All poles of $\mathbb{K}(\xi, x)$ and $\mathbb{L}(\xi, x)$ are supposed to be simple, and the empty product is treated as unity.

$$
\begin{gathered}
\mathfrak{H}_{i}>0, \quad|\arg (z)|<\frac{\pi}{2} \mathfrak{H}_{i}, \quad i=\overline{1, r}, \\
\mathfrak{H}_{i} \geq 0, \quad|\arg (z)|<\frac{\pi}{2} \mathfrak{H}_{i} \quad \text { and } \quad \mathfrak{R}\left(\zeta_{i}\right)+1<0,
\end{gathered}
$$

where:

$$
\begin{gathered}
\mathfrak{H}_{i}=\sum_{j=2}^{n} E_{j}+\sum_{j=2}^{m} F_{j}-\sum_{j=n+1}^{p_{i}} E_{j i}-\sum_{j=m+1}^{q_{i}} F_{j i}, \\
\zeta_{i}=\sum_{j=2}^{m} f_{j}-\sum_{j=2}^{n} e_{j}+\sum_{j=m+1}^{q_{i}} E_{j i}-\sum_{j=n+1}^{p_{i}} F_{j i}+\frac{1}{2}\left(p_{i}-q_{i}\right), \quad i=\overline{1, r} .
\end{gathered}
$$

The incomplete I-functions ${ }^{(\Gamma)} I_{p_{\ell, q_{\ell}, r}}^{m, n}(z)$ and ${ }^{(\gamma)} I_{p_{\ell, q_{\ell}, r}}^{m, n}(z)$ presented in (11) and (13) reduce to the many well-known special functions as follows:

1. On setting $x=0,(11)$ and (13) reduce to the $I$-function proposed by Saxena [30]:

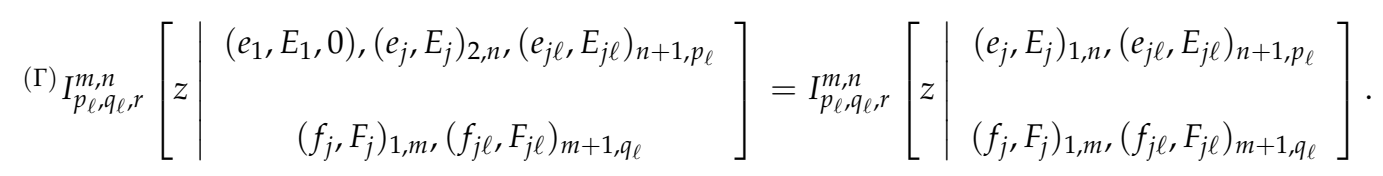

2. Again, setting $r=1$ in (11) and (13), then it reduces to the IHFs introduced by Srivastava [31] (see also [32]):

$$
{ }^{(\Gamma)} I_{p_{\ell}, q_{\ell}, 1}^{m, n}\left[z \mid \begin{array}{c}
\left(e_{1}, E_{1}, x\right),\left(e_{j}, E_{j}\right)_{2, n},\left(e_{j \ell}, E_{j \ell}\right)_{n+1, p_{\ell}} \\
\left(f_{j}, F_{j}\right)_{1, m},\left(f_{j \ell}, F_{j \ell}\right)_{m+1, q_{\ell}}
\end{array}\right]=\Gamma_{p, q}^{m, n}\left[\begin{array}{c}
z \mid\left(e_{1}, E_{1}, x\right),\left(e_{j}, E_{j}\right)_{2, p} \\
\left(f_{j}, F_{j}\right)_{1, q}
\end{array}\right],
$$

and:

$$
{ }^{(\gamma)} I_{p_{\ell, q_{\ell}, 1}^{m, n}}^{m}\left[\begin{array}{c}
\left(e_{1}, E_{1}, x\right),\left(e_{j}, E_{j}\right)_{2, n},\left(e_{j \ell}, E_{j \ell}\right)_{n+1, p_{\ell}} \\
\left(f_{j}, F_{j}\right)_{1, m},\left(f_{j \ell}, F_{j \ell}\right)_{m+1, q_{\ell}}
\end{array}\right]=\gamma_{p, q}^{m, n}\left[\begin{array}{c}
\left(e_{1}, E_{1}, x\right)_{,},\left(e_{j}, E_{j}\right)_{2, p} \\
\left(f_{j}, F_{j}\right)_{1, q}
\end{array}\right] .
$$

A complete description of IHFs can be found in the article [31].

3. Further, taking $m=1, n=p_{\ell}, q_{\ell}$ is replaced by $q_{\ell}+1$, and taking the suitable parameter, then the functions (20) and (21) reduce to the incomplete Fox-Wright $\Psi$-functions $\Psi_{q}^{(\Gamma)}$ and $\Psi_{p} \Psi_{q}^{(\gamma)}$, which were defined by Srivastava et al. [31].

$$
\Gamma_{p, q+1}^{1, p}\left[-z \mid \begin{array}{c}
\left(1-e_{1}, E_{1}, x\right),\left(1-e_{j}, E_{j}\right)_{2, p} \\
(0,1),\left(1-f_{j}, F_{j}\right)_{1, q}
\end{array}\right]={ }_{p} \Psi_{q}^{(\Gamma)}\left[\begin{array}{c}
\left(e_{1}, E_{1}, x\right),\left(e_{j}, E_{j}\right)_{2, p} ; \\
\left(f_{j}, F_{j}\right)_{1, q} ;
\end{array}\right]
$$

and:

$$
\gamma_{p, q+1}^{1, p}\left[-z \mid \begin{array}{c}
\left(1-e_{1}, E_{1}, x\right),\left(1-e_{j}, E_{j}\right)_{2, p} \\
(0,1),\left(1-f_{j}, F_{j}\right)_{1, q}
\end{array}\right]={ }_{p} \Psi_{q}^{(\gamma)}\left[\begin{array}{c}
\left(e_{1}, E_{1}, x\right),\left(e_{j}, E_{j}\right)_{2, p} ; \\
\left(f_{j}, F_{j}\right)_{1, q} ;
\end{array}\right]
$$


4. Next, we take $x=0$ and $r=1$ in (11). The incomplete $I$-function reduces to the familiar Fox's $H$-function, which is defined and expressed in the following manner (see, for example, [33], p. 10):

$$
{ }^{(\Gamma)} I_{p_{\ell}, q_{\ell}, 1}^{m, n}\left[z\left[\begin{array}{c}
\left(e_{1}, E_{1}, 0\right),\left(e_{j}, E_{j}\right)_{2, n},\left(e_{j \ell}, E_{j \ell}\right)_{n+1, p_{\ell}} \\
\left(f_{j}, F_{j}\right)_{1, m},\left(f_{j \ell}, F_{j \ell}\right)_{m+1, q_{\ell}}
\end{array}\right]=H_{p, q}^{m, n}\left[\begin{array}{c}
\left(e_{1}, E_{1}\right), \cdots,\left(e_{p}, E_{p}\right) \\
\left(f_{1}, F_{1}\right), \cdots,\left(f_{q}, F_{q}\right)
\end{array}\right] .\right.
$$

Numerous special functions can be obtained from the incomplete $I$-functions for which some interesting functions are used in Section 3.

\section{Arbitrary-Order Kinetic Equation}

This part deals with the solution of FKE associated with the incomplete I-functions (11) and (13).

Theorem 1. If $x>0, \alpha>0, \beta>0, c>0, \wp>0, E_{l}>0(\imath=1, \cdots, p)$, and $F_{l}>0(\imath=1, \cdots, q)$, then the following arbitrary-order kinetic equation:

$$
\Theta(w)-\Theta_{0} w^{\alpha-1(\Gamma)} I_{p_{\ell}, q_{\ell}, r}^{m, n}\left[-\wp^{\beta} w^{\beta} \mid \begin{array}{c}
\left(e_{1}, E_{1}, x\right),\left(e_{l}, E_{l}\right)_{2, n},\left(e_{l \ell}, E_{l \ell}\right)_{n+1, p_{\ell}} \\
\left(f_{l}, F_{l}\right)_{1, m},\left(f_{\ell \ell}, F_{l \ell}\right)_{m+1, q_{\ell}}
\end{array}\right]=-c^{\beta}{ }_{0} D_{w}^{-\beta} \Theta(w),
$$

has a solution of the form:

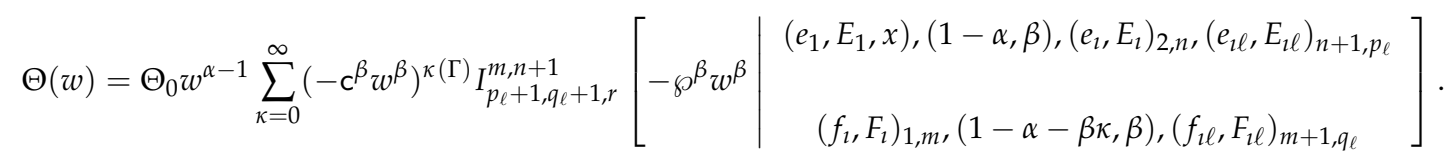

Proof. Taking the Laplace transform [34] on both sides of FKE (25), we obtain:

$$
\bar{\Theta}(\mathfrak{p})-\Theta_{0} \frac{1}{2 \pi i} \int_{\mathfrak{L}} \mathbb{K}(\xi, x)\left(-\wp^{\beta}\right)^{-\zeta} \frac{\Gamma(\alpha-\beta \xi)}{\mathfrak{p}^{\alpha-\beta \xi}} d \xi=-c^{\beta} \mathfrak{p}^{-\beta} \bar{\Theta}(\mathfrak{p}),
$$

where $\mathbb{K}(\xi, x)$ is given in (12).

Upon simplifying the above equation, we get:

$$
\begin{aligned}
\bar{\Theta}(\mathfrak{p}) & =\frac{\Theta_{0}}{\left(1+c^{\beta} \mathfrak{p}^{-\beta}\right)} \frac{1}{2 \pi i} \int_{\mathfrak{L}} \mathbb{K}(\xi, x)\left(-\wp^{\beta}\right)^{-\xi} \frac{\Gamma(\alpha-\beta \xi)}{\mathfrak{p}^{\alpha-\beta \xi}} d \xi, \\
& =\Theta_{0} \sum_{\kappa=0}^{\infty}(-1)^{\kappa}\left(c^{\beta} \mathfrak{p}^{-\beta}\right)^{\kappa} \frac{1}{2 \pi i} \int_{\mathfrak{L}} \mathbb{K}(\xi, x)\left(-\wp^{\beta}\right)^{-\xi} \frac{\Gamma(\alpha-\beta \xi)}{\mathfrak{p}^{\alpha-\beta \xi}} d \xi .
\end{aligned}
$$

Finally, taking the inverse Laplace transform on both sides of the trailing equation, we get the desired result (26).

Theorem 2. If $x>0, \alpha>0, \beta>0, c>0, \wp>0, E_{\imath}>0(\imath=1, \cdots, p)$, and $F_{l}>0(\imath=1, \cdots, q)$, then the following arbitrary-order kinetic equation:

$$
\Theta(w)-\Theta_{0} w^{\alpha-1(\gamma)} I_{p_{\ell}, q_{\ell}, r}^{m, n}\left[\begin{array}{c|c}
\left(e_{1}, E_{1}, x\right),\left(e_{l}, E_{l}\right)_{2, n},\left(e_{l \ell}, E_{l \ell}\right)_{n+1, p_{\ell}} \\
\left(f_{l}, F_{l}\right)_{1, m},\left(f_{l \ell}, F_{l \ell}\right)_{m+1, q_{\ell}}
\end{array}\right]=-c^{\beta}{ }_{0} D_{w}^{-\beta} \Theta(w),
$$


has a solution of the form:

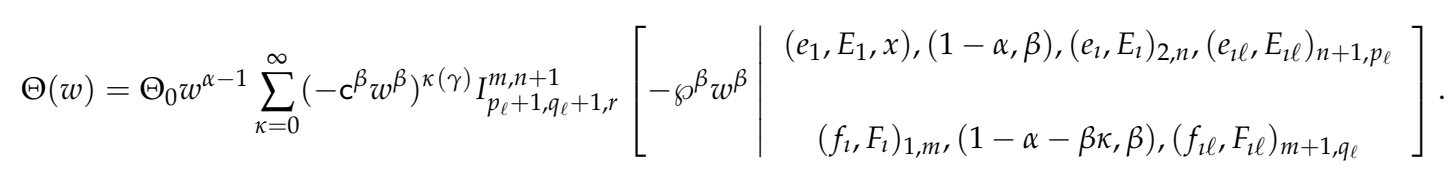

Proof. The proof of Theorem 2 is given in a similar way as that of Theorem 1.

\section{Special Cases and Remarks}

In this part, we record certain interesting corollaries of the main results (Theorems 1 and 2):

Corollary 1. If $\alpha>0, \beta>0, c>0, \wp>0, E_{\imath}>0(\imath=1, \cdots, p)$, and $F_{l}>0(\imath=1, \cdots, q)$, then the following arbitrary-order kinetic equation:

$$
\Theta(w)-\Theta_{0} w^{\alpha-1} I_{p_{\ell}, q_{\ell}, r}^{m, n}\left[-\gamma^{\beta} w^{\beta} \mid \begin{array}{c}
\left(e_{l}, E_{l}\right)_{1, n},\left(e_{\imath \ell}, E_{\imath \ell}\right)_{n+1, p_{\ell}} \\
\left(f_{l}, F_{l}\right)_{1, m},\left(f_{\ell \ell}, F_{\imath \ell}\right)_{m+1, q_{\ell}}
\end{array}\right]=-c^{\beta}{ }_{0} D_{w}^{-\beta} \Theta(w),
$$

has a solution of the form:

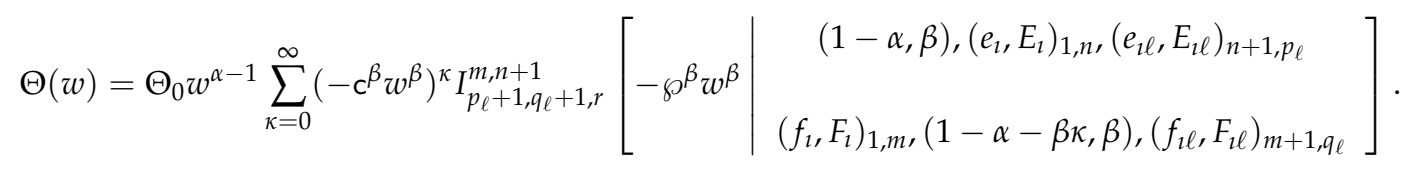

Proof. Taking $x=0$ in the result (25), we get the desired result.

Corollary 2. If $x>0, \alpha>0, \beta>0, c>0, \wp>0, E_{l}>0(\imath=1, \cdots, p)$, and $F_{l}>0(\imath=1, \cdots, q)$, then the following arbitrary-order kinetic equation:

$$
\Theta(w)-\Theta_{0} w^{\alpha-1} \Gamma_{p, q}^{m, n}\left[-\wp^{\beta} w^{\beta} \mid \begin{array}{c}
\left(e_{1}, E_{1}, x\right),\left(e_{l}, E_{l}\right)_{2, p} \\
\left(f_{l}, F_{l}\right)_{1, q}
\end{array}\right]=-c^{\beta}{ }_{0} D_{w}^{-\beta} \Theta(w),
$$

has a solution of the form:

$$
\Theta(w)=\Theta_{0} w^{\alpha-1} \sum_{\kappa=0}^{\infty}\left(-c^{\beta} w^{\beta}\right)^{\kappa} \Gamma_{p+1, q+1}^{m, n+1}\left[\begin{array}{c|c}
-\wp^{\beta} w^{\beta} & \left(e_{1}, E_{1}, x\right),(1-\alpha, \beta),\left(e_{l}, E_{l}\right)_{2, p} \\
\left(f_{l}, F_{l}\right)_{1, q},(1-\alpha-\beta \kappa, \beta)
\end{array}\right] .
$$

Proof. Again, setting $r=1$ in Theorem 1, we achieve the desired result (32).

Corollary 3. If $x>0, \alpha>0, \beta>0, c>0, \wp>0, E_{\imath}>0(\imath=1, \cdots, p)$, and $F_{\imath}>0(\imath=1, \cdots, q)$, then the following arbitrary-order kinetic equation:

$$
\Theta(w)-\Theta_{0} w^{\alpha-1} \gamma_{p, q}^{m, n}\left[-\wp^{\beta} w^{\beta} \mid \begin{array}{c}
\left(e_{1}, E_{1}, x\right),\left(e_{l}, E_{l}\right)_{2, p} \\
\left(f_{l}, F_{l}\right)_{1, q}
\end{array}\right]=-c^{\beta}{ }_{0} D_{w}^{-\beta} \Theta(w),
$$


has a solution of the form:

$$
\Theta(w)=\Theta_{0} w^{\alpha-1} \sum_{\kappa=0}^{\infty}\left(-c^{\beta} w^{\beta}\right)^{\kappa} \gamma_{p+1, q+1}^{m, n+1}\left[\begin{array}{c|c}
-\gamma^{\beta} w^{\beta} & \left(e_{1}, E_{1}, x\right),(1-\alpha, \beta),\left(e_{l}, E_{l}\right)_{2, p} \\
\left(f_{l}, F_{l}\right)_{1, q},(1-\alpha-\beta \kappa, \beta)
\end{array}\right] .
$$

Proof. Setting $r=1$ in Theorem 2, we arrive at the desired result (34).

Corollary 4. If $x>0, \alpha>0, \beta>0, c>0, \wp>0, E_{\imath}>0(\imath=1, \cdots, p)$, and $F_{l}>0(\imath=1, \cdots, q)$, then the following arbitrary-order kinetic equation:

$$
\Theta(w)-\Theta_{0} w^{\alpha-1}{ }_{p} \psi_{q}^{(\Gamma)}\left[\begin{array}{c|c}
\gamma^{\beta} w^{\beta} & \left(e_{1}, E_{1}, x\right),\left(e_{l}, E_{l}\right)_{2, p} \\
\left(f_{l}, F_{l}\right)_{1, q}
\end{array}\right]=-c^{\beta}{ }_{0} D_{w}^{-\beta} \Theta(w),
$$

has a solution of the form:

$$
\Theta(w)=\Theta_{0} w^{\alpha-1} \sum_{\kappa=0}^{\infty}\left(-c^{\beta} w^{\beta}\right)^{\kappa}{ }_{p+1} \Psi_{q+1}^{(\Gamma)}\left[\begin{array}{l|c}
\wp^{\beta} w^{\beta} & \left(e_{1}, E_{1}, x\right),(1-\alpha, \beta),\left(e_{l}, E_{l}\right)_{2, p} \\
\left(f_{l}, F_{l}\right)_{1, q},(1-\alpha-\beta \kappa, \beta)
\end{array}\right] .
$$

Proof. Taking the suitable parameter in Equation (31), we get the desired result.

Corollary 5. If $x>0, \alpha>0, \beta>0, c>0, \wp>0, E_{\imath}>0(\imath=1, \cdots, p)$, and $F_{l}>0(\imath=1, \cdots, q)$, then the following arbitrary-order kinetic equation:

$$
\Theta(w)-\Theta_{0} w^{\alpha-1}{ }_{p} \Psi_{q}^{(\gamma)}\left[\begin{array}{c|c}
\gamma^{\beta} w^{\beta} & \left(e_{1}, E_{1}, x\right),\left(e_{l}, E_{l}\right)_{2, p} \\
\left(f_{l}, F_{l}\right)_{1, q}
\end{array}\right]=-c^{\beta}{ }_{0} D_{w}^{-\beta} \Theta(w),
$$

has a solution of the form:

$$
\Theta(w)=\Theta_{0} w^{\alpha-1} \sum_{\kappa=0}^{\infty}\left(-c^{\beta} w^{\beta}\right)^{\kappa}{ }_{p+1} \psi_{q+1}^{(\gamma)}\left[\begin{array}{c|c}
\gamma^{\beta} w^{\beta} & \left(e_{1}, E_{1}, x\right),(1-\alpha, \beta),\left(e_{l}, E_{l}\right)_{2, p} \\
\left(f_{l}, F_{l}\right)_{1, q},(1-\alpha-\beta \kappa, \beta)
\end{array}\right]
$$

Proof. Taking the suitable parameter in Equation (33), we get the desired result.

Corollary 6. If $\alpha>0, \beta>0, c>0, \wp>0, E_{l}>0(\imath=1, \cdots, p)$, and $F_{l}>0(\imath=1, \cdots, q)$, then the following arbitrary-order kinetic equation:

$$
\Theta(w)-\Theta_{0} w^{\alpha-1} H_{p, q}^{m, n}\left[-\wp^{\beta} w^{\beta} \mid \begin{array}{c}
\left(e_{l}, E_{l}\right)_{1, p} \\
\left(f_{l}, F_{l}\right)_{1, q}
\end{array}\right]=-c^{\beta}{ }_{0} D_{w}^{-\beta} \Theta(w),
$$

has a solution of the form:

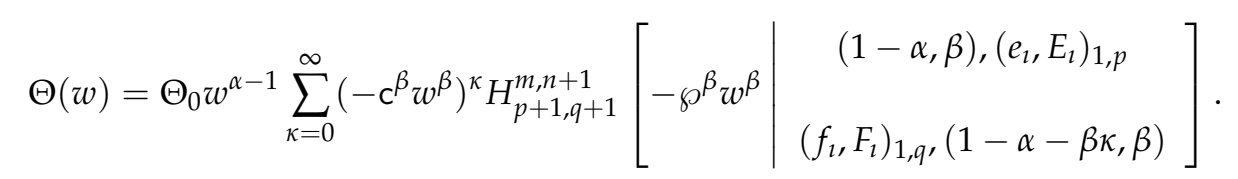

Proof. Again, taking $x=0$ in (31), we achieve the required result. 
Corollary 7. If $\alpha>0, \beta>0, c>0, \wp>0, E_{l}>0(\imath=1, \cdots, p)$, and $F_{l}>0(\imath=1, \cdots, q)$, then the following fractional kinetic equation:

$$
\Theta(w)-\Theta_{0} w^{\alpha-1} \Psi_{q}\left[\wp^{\beta} w^{\beta} \mid \begin{array}{c}
\left(e_{1}, E_{l}\right)_{1, p} \\
\left(f_{l}, F_{l}\right)_{1, q}
\end{array}\right]=-c^{\beta}{ }_{0} D_{w}^{-\beta} \Theta(w),
$$

has a solution of the form:

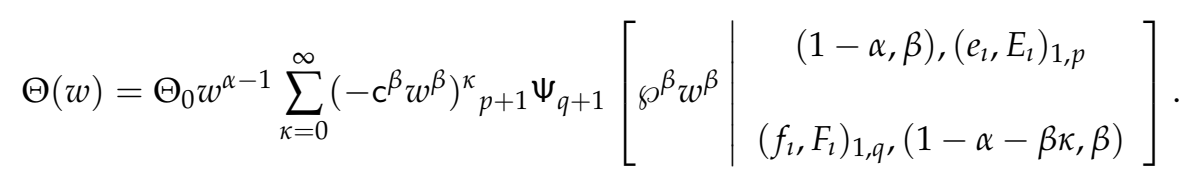

Proof. Again, taking $x=0$ in (35) and (37), we arrive at the required result.

Corollary 8. If $x>0, \alpha>0, \beta>0, c>0, \wp>0$, then the following arbitrary-order kinetic equation:

$$
\Theta(w)-\Theta_{0} w^{\alpha-1}{ }_{p} \Gamma_{q}\left[\gamma_{\gamma^{\beta}} w^{\beta} \mid \begin{array}{c}
\left(e_{1}, x\right), e_{2}, \cdots, e_{p} \\
f_{1}, \cdots, f_{q}
\end{array}\right]=-c^{\beta}{ }_{0} D_{w}^{-\beta} \Theta(w),
$$

has a solution of the form:

$$
\Theta(w)=\Theta_{0} w^{\alpha-1} \sum_{\kappa=0}^{\infty}\left(-c^{\beta} w^{\beta}\right)^{\kappa} \Gamma_{p+1, q+2}^{1, p+1}\left[\wp^{\beta} w^{\beta} \mid \begin{array}{c}
\left(1-e_{1}, 1, x\right),(\alpha, \beta),\left(1-e_{l}, 1\right)_{2, p} \\
(0,1),\left(1-f_{l}, 1\right)_{1, q},(\alpha+\beta \kappa, \beta)
\end{array}\right] .
$$

Proof. Again, setting $E_{\imath}=F_{k}=1(\imath=1, \cdots, p ; k=1, \cdots, q)$ in Equation (31), we arrive at the desired result (44).

Corollary 9. If $x>0, \alpha>0, \beta>0, c>0, \wp>0$, then the following arbitrary-order kinetic expression:

$$
\Theta(w)-\Theta_{0} w^{\alpha-1}{ }_{p} \gamma_{q}\left[\wp_{\wp}^{\beta} w^{\beta} \mid \begin{array}{c}
\left(e_{1}, x\right), e_{2}, \cdots, e_{p} \\
f_{1}, \cdots, f_{q}
\end{array}\right]=-c^{\beta}{ }_{0} D_{w}^{-\beta} \Theta(w),
$$

has a solution of the form:

$$
\Theta(w)=\Theta_{0} w^{\alpha-1} \sum_{\kappa=0}^{\infty}\left(-c^{\beta} w^{\beta}\right)^{\kappa} \gamma_{p+1, q+2}^{1, p+1}\left[\wp^{\beta} w^{\beta} \mid \begin{array}{c}
\left(1-e_{1}, 1, x\right),(\alpha, \beta),\left(1-e_{l}, 1\right)_{2, p} \\
(0,1),\left(1-f_{l}, 1\right)_{1, q},(\alpha+\beta \kappa, \beta)
\end{array}\right] .
$$

Proof. Again, setting $E_{\imath}=F_{k}=1(\imath=1, \cdots, p ; k=1, \cdots, q)$ in Equation (33), we arrive at the desired result (46).

Corollary 10. If $\alpha>0, \beta>0, c>0, \wp>0$, then the following arbitrary-order kinetic expression:

$$
\Theta(w)-\Theta_{0} w^{\alpha-1}{ }_{p} F_{q}\left[\wp_{\wp} w^{\beta} \mid \begin{array}{c}
e_{1}, \cdots, e_{p} \\
f_{1}, \cdots, f_{q}
\end{array}\right]=-c^{\beta}{ }_{0} D_{w}^{-\beta} \Theta(w),
$$


has a solution of the form:

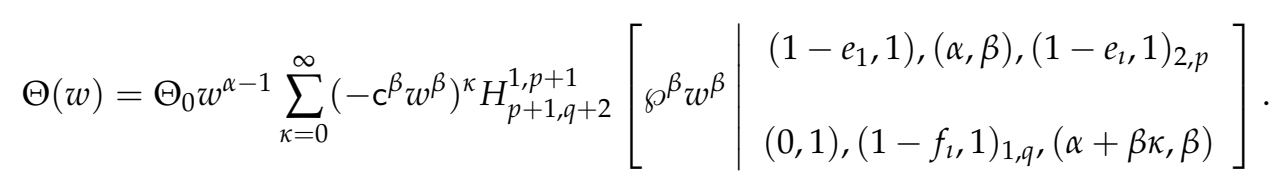

Proof. Again, setting $x=0$ in Equation (43), we arrive at the desired result (48).

Remark 1. If Fox's H-function reduces to generalized $M$-series ${ }_{p}^{\alpha, \beta} M_{q}\left(a_{1}, \cdots, a_{p} ; b_{1}, \cdots, b_{q} ; z\right)$ in Equation (39), then the result is that recorded by Chaurasia and Kumar ([35], p. 777, Equation (14)).

Remark 2. If the Fox-Wright function ${ }_{p} \Psi_{q}(z)$ reduces to the Mittag-Leffler function $E_{\alpha, \beta}(z)$ in Equation (41), then the result is that recorded by Saxena et al. ([15], Equation (21)).

Remark 3. If the incomplete H-function reduces to the Bessel function of the first kind $J_{v}(z)$ in (31), then the result is that recorded by Habenom et al. [12].

\section{Numerical Results and Discussion}

In this section, we simulate the numerical results for FKE (25) at different values of various parameters presented in the form of Figures 1 and 2 by using Maple. We can see from Figures 1 and 2 that the value of $\Theta$ decreases with time $w$. It is also noticed from Figures 1 and 2 that as the value of $\beta$ increases, the corresponding value of $\Theta$ initially enhances, but after some time, it depicts the opposite nature.

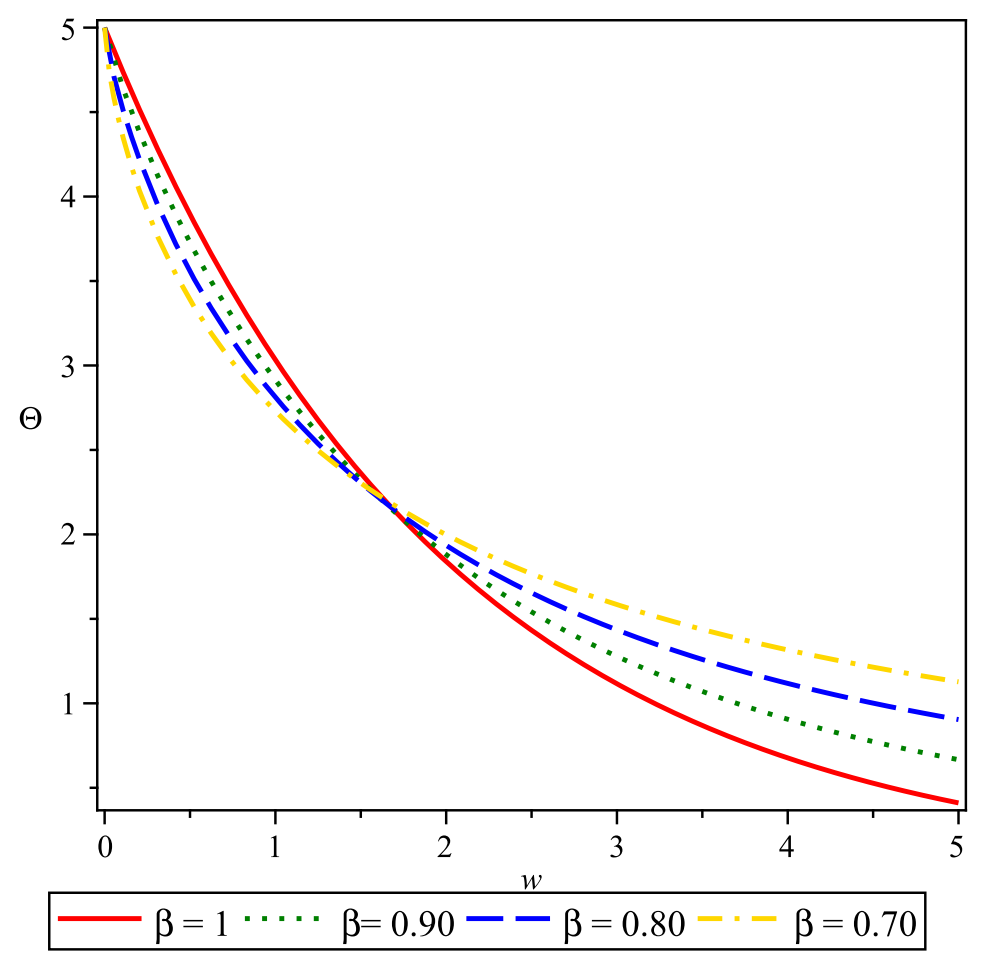

Figure 1. Plots of solution $\Theta$ for the fractional kinetic equation (FKE) (25) when $\Theta_{0}=5, \wp=0$, and $\mathrm{c}=0.5$. 


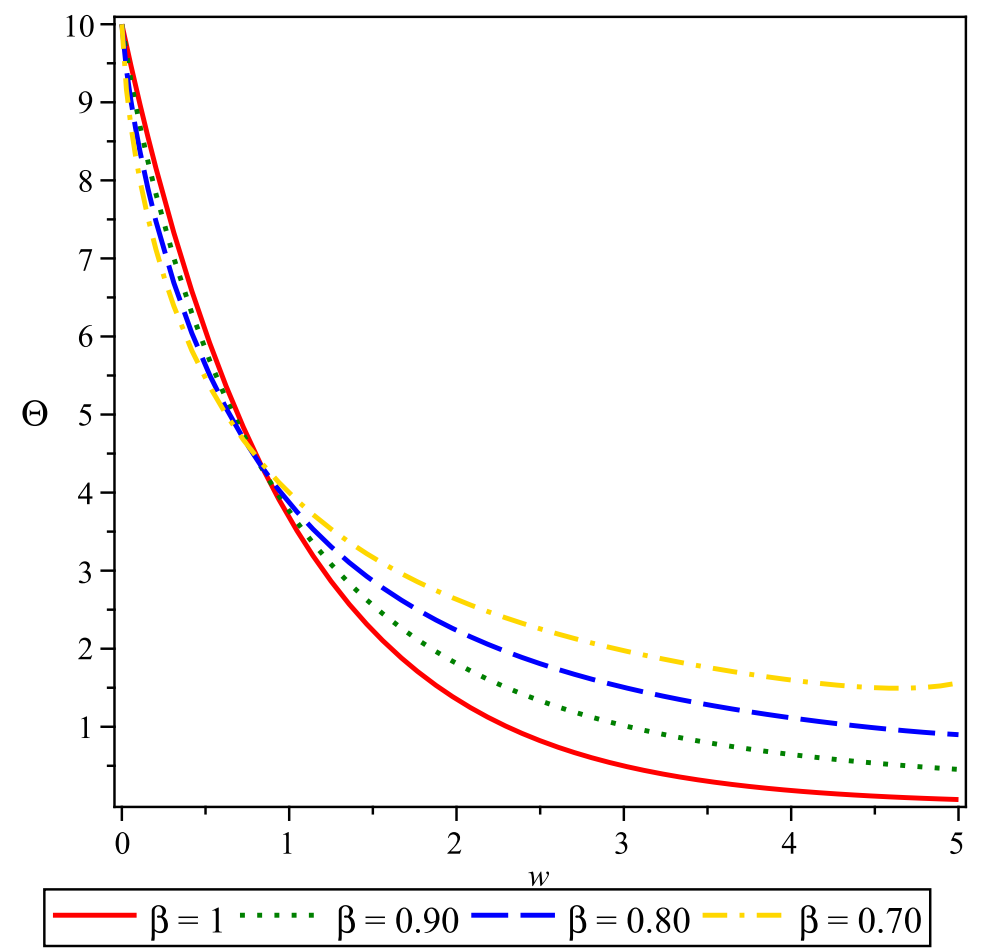

Figure 2. Plots of solution $\Theta$ for FKE (25) when $\Theta_{0}=10, \wp=0$, and $c=1$.

\section{Conclusions}

In this work, we introduced generalized FKEs of the FKE associated with the incomplete $I$-functions and found their solutions in terms of incomplete $I$-functions. The novelty and importance of the present work were that we suggested a novel computable extension of FKEs in terms of incomplete $I$-functions and presented some numerical results in graphical form, which were very useful to study reaction rate. The FKEs could be employed to determine the particle reaction rate and interpret the statistical mechanics pertaining to the particle distribution function. We also derived some special cases by assigning particular values to the parameters of incomplete $I$-functions and also provided some known and important results. The outcomes of the present study are very useful in astrophysics and space science.

Author Contributions: Conceptualization, M.K.B. and D.K.; methodology, M.K.B. and J.S.; software, M.K.B. and D.K.; validation, D.K., P.H., and J.S.; formal analysis, P.H. and J.S.; investigation, M.K.B. and D.K.; resources, M.K.B.; data curation, D.K.; writing, original draft preparation, M.K.B. and J.S.; writing, review and editing, P.H. and J.S.; visualization, D.K.; supervision, D.K. and J.S.; project administration, M.K.B.; funding acquisition, M.K.B. All authors read and agreed to the published version of the manuscript.

Funding: The present investigation was supported, in part, by the TEQIP-III under CRS Grant 1-5730065311.

Acknowledgments: The authors would like to express their deep-felt thanks for the reviewers' valuable comments to improve this paper as it stands.

Conflicts of Interest: The authors declare no conflict of interest.

\section{References}

1. Miller, K. S.; Ross, B. An Introduction to the Fractional Calculus and Fractional Differential Equations; John Wiley \& Sons, INC.: New York, NY, USA, 1993.

2. Oldham, K.; Spanier, J. Fractional Calculus: Theory and Applications of Differentiation and Integration of Arbitrary Order; Academic Press: New York, NY, USA, 1974. 
3. Kilbas, A.A.; Srivastava, H.M.; Trujillo, J.J. Theory and Applications of Fractional Differential Equations; North-Holland Mathematical Studies; Elsevier (North-Holland) Science Publishers: Amsterdam, The Netherlands; London, UK; New York, NY, USA, 2006; Volume 204.

4. Podlubny, I. Fractional Differential Equations; Academic Press: San Diego, CA, USA, 1999.

5. Srivastava, H.M.; Saxena, R.K. Operators of Fractional Integration and Their Applications. Appl. Math. Comput. 2001, 118, 1-52. [CrossRef]

6. Haubold, H.J.; Mathai, A.M. The fractional kinetic equation and thermonuclear functions. Astrophys. Space Sci. 2000, 273, 53-63. [CrossRef]

7. Saichev, A.I.; Zaslavsky, G.M. Fractional kinetic equations: Solutions and applications. Chaos 1997, 7, $753-764$. [CrossRef] [PubMed]

8. Saxena, R.K.; Mathai, A.M.; Haubold, H.J. On generalized fractional kinetic equations. Physics A 2004, 344, 657-664. [CrossRef]

9. Saxena, R.K.; Kalla, S.L. On the solutions of certain fractional kinetic equations. Appl. Math. Comput. 2008, 199, 504-511. [CrossRef]

10. Chaurasia, V.B.L.; Pandey, S.C. On the new computable solution of the generalized fractional kinetic equations involving the generalized function for the fractional calculus and related functions. Astrophys. Space Sci. 2008, 317, 213-219. [CrossRef]

11. Goswami, A.; Singh, J.; Kumar, D.; Sushila. An efficient analytical approach for fractional equal width equations describing hydro-magnetic waves in cold plasma. Physics A 2019, 524, 563-575. [CrossRef]

12. Habenom, H.; Suthar, D.L.; Gebeyehu, M. Application of Laplace Transform on Fractional Kinetic Equation Pertaining to the Generalized Galué Type Struve Function. Adv. Math. Phys. 2019, 2019, 1-8. [CrossRef]

13. Kumar, D.; Singh, J.; Tanwar, K.; Baleanu, D. A new fractional exothermic reactions model having constant heat source in porous media with power, exponential and Mittag-Leffler Laws. Int. J. Heat Mass Transf. 2019, 138, 1222-1227. [CrossRef]

14. Kumar, D.; Singh, J.; Qurashi, M.A.; Baleanu, D. A new fractional SIRS-SI malaria disease model with application of vaccines, anti-malarial drugs, and spraying. Adv. Differ. Equ. 2019, 2019, 278. [CrossRef]

15. Saxena, R.K.; Mathai, A.M.; Haubold, H.J. On Fractional Kinetic Equations. Astrophys. Space Sci. 2002, 282, 281-287. [CrossRef]

16. Yavuz, M.; Özdemir, N. Numerical inverse Laplace homotopy technique for fractional heat equations. Therm. Sci. 2018, 22, 185-194. [CrossRef]

17. Yavuz, M.; Özdemir, N. European vanilla option pricing model of fractional order without singular kernel. Fractal Fract. 2018, 2, 3. [CrossRef]

18. Abramowitz, M.; Stegun, I.A. Handbook of Mathematical Functions with Formulas; Graphs; and Mathematical Tables, Applied Mathematics Series; National Bureau of Standards: Washington, DC, USA, 1964; Dover Publications: New York, NY, USA, 1965; Volume 55.

19. Andrews, L.C. Special Functions for Engineers and Applied Mathematicians; Macmillan Company: New York, NY, USA, 1985.

20. Bansal, M.K.; Kumar, D.; Jain, R. Interrelationships Between Marichev-Saigo-Maeda Fractional Integral Operators, the Laplace Transform and the $\bar{H}$-Function. Int. J. Appl. Comput. Math 2019, 5, 103. [CrossRef]

21. Bansal, M.K.; Jolly, N.; Jain, R.; Kumar, D. An integral operator involving generalized Mittag-Leffler function and associated fractional calculus results. J. Anal. 2019, 27, 727-740. [CrossRef]

22. Bansal, M.K.; Kumar, D.; Jain, R. A Study of Marichev-Saigo-Maeda Fractional Integral Operators Associated with S-Generalized Gauss Hypergeometric Function. Kyungpook Math. J. 2019, 59, 433-443.

23. Goswami, A.; Singh, J.; Kumar, D.; Gupta, S.; Sushila. An efficient analytical technique for fractional partial differential equations occurring in ion acoustic waves in plasma. J. Ocean Eng. Sci. 2019, 4, 85-99. [CrossRef]

24. Kumar, D.; Singh, J.; Baleanu, D. On the analysis of vibration equation involving a fractional derivative with Mittag-Leffler law. Math. Methods Appl. Sci. 2020, 43, 443-457. [CrossRef]

25. Kumar, D.; Singh, J.; Purohit, S.D.; Swroop, R. A hybrid analytical algorithm for nonlinear fractional wave-like equations. Math. Model. Nat. Phenom. 2019, 14, 304. [CrossRef]

26. Singh, J.; Kumar, D.; Baleanu, D. New aspects of fractional Biswas-Milovic model with Mittag-Leffler law. Math. Model. Nat. Phenom. 2019, 14, 303. [CrossRef] 
27. Srivastava, H.M.; Chaudhry, M.A.; Agarwal, R.P. The incomplete Pochhammer symbols and their applications to hypergeometric and related functions. Integral Transform. Spec. Funct. 2012, 23, 659-683. [CrossRef]

28. Srivastava, H.M.; Bansal, M.K.; Harjule, P. A study of fractional integral operators involving a certain generalized multi-index Mittag-Leffler function. Math. Methods Appl. Sci. 2018, 41, 6108-6121. [CrossRef]

29. Bansal, M.K.; Kumar, D. On the integral operators pertaining to a family of incomplete I-functions. AIMS Math. 2020, 5, 1247-1259. [CrossRef]

30. Saxena, V.P. Formal solution of certain new pair of dual integral equations involving H-functions. Proc. Natl. Acad. Sci. India Sect. A 1982, 52, 366-375.

31. Srivastava, H.M.; Saxena, R.K.; Parmar, R.K. Some Families of the Incomplete H-Functions and the Incomplete $\bar{H}$-Functions and Associated Integral Transforms and Operators of Fractional Calculus with Applications. Russ. J. Math. Phys. 2018, 25, 116-138. [CrossRef]

32. Bansal, M.K.; Choi, J. A Note on Pathway Fractional Integral Formulas Associated with the Incomplete H-Functions. Int. J. Appl. Comput. Math. 2019, 5, 133. [CrossRef]

33. Srivastava, H.M.; Gupta, K.C.; Goyal, S.P. The H-Functions of One and Two Variables with Applications; South Asian Publishers: New Delhi, India, 1982.

34. Sneddon, I.N. The Use of Integral Transforms; Tata McGrawHill: New Delhi, India, 1979.

35. Chaurasia, V.B.L.; Kumar, D. On the solutions of generalized fractional kinetic equations. Adv. Stud. Theor. Phys. 2010, 4, 773-780.

(C) 2020 by the authors. Licensee MDPI, Basel, Switzerland. This article is an open access article distributed under the terms and conditions of the Creative Commons Attribution (CC BY) license (http://creativecommons.org/licenses/by/4.0/). 